\title{
COP9 signalosome subunit 5A affects phenylpropanoid metabolism, trichome formation and transcription of key genes of a regulatory tri-protein complex in Arabidopsis
}

\author{
Shu Wei ${ }^{1,2^{*}}\left(\mathbb{D}\right.$, Xiang Li $i^{2,3}$, Margaret Y. Gruber ${ }^{2}$, Biruk A. Feyissa ${ }^{4}$, Lisa Amyot ${ }^{4}$ and Abdelali Hannoufa ${ }^{4^{*}}$
}

\begin{abstract}
Background: Trichomes and phenylpropanoid-derived phenolics are structural and chemical protection against many adverse conditions. Their production is regulated by a network that includes a TTG1/bHLH/MYB tri-protein complex in Arabidopsis. CSN5a, encoding COP9 signalosome subunit 5a, has also been implicated in trichome and anthocyanin production; however, the regulatory roles of CSN5a in the processes through interaction with the triprotein complex has yet to be investigated.

Results: In this study, a new csn5a mutant, sk372, was recovered based on its altered morphological and chemical phenotypes compared to wild-type control. Mutant characterization was conducted with an emphasis on trichome and phenylpropanoid production and possible involvement of the tri-protein complex using metabolite and gene transcription profiling and scanning electron microscopy. Seed metabolite analysis revealed that defective CSN5a led to an enhanced production of many compounds in addition to anthocyanin, most notably phenylpropanoids and carotenoids as well as a glycoside of zeatin. Consistent changes in carotenoids and anthocyanin were also found in the $s k 372$ leaves. In addition, 370 genes were differentially expressed in 10-day old seedlings of $s k 372$ compared to its wild type control. Real-time transcript quantitative analysis showed that in sk372, GL2 and triprotein complex gene TT2 was significantly suppressed $(p<0.05)$ while complex genes EGL3 and GL3 slightly decreased $(p>0.05)$. Complex genes MYB75, GL1 and flavonoid biosynthetic genes $\Pi 3$ and $T 18$ in sk372 were all significantly enhanced. Overexpression of GL3 driven by cauliflower mosaic virus 355 promotor increased the number of single pointed trichomes only, no other phenotypic recovery in sk372.

Conclusions: Our results indicated clearly that COP9 signalosome subunit CSN5a affects trichome production and the metabolism of a wide range of phenylpropanoid and carotenoid compounds. Enhanced anthocyanin accumulation and reduced trichome production were related to the enhanced MYB75 and suppressed GL2 and some other differentially expressed genes associated with the TTG1/bHLH/MYB complexes.
\end{abstract}

Keywords: CSN5a, Arabidopsis, COP9 signalosome, Anthocyanin, Trichomes, MYB75

\footnotetext{
* Correspondence: weishu@ahau.edu.cn; abdelali.hannoufa@agr.gc.ca

${ }^{1}$ State Key Laboratory of Tea Plant Biology and Utilization, Anhui Agricultural University, Hefei, Anhui, China

${ }^{4}$ Agriculture and Agri-Food Canada and Department of Biology, University of

Western Ontario, London, ON, Canada

Full list of author information is available at the end of the article
}

(c) The Author(s). 2018 Open Access This article is distributed under the terms of the Creative Commons Attribution 4.0 International License (http://creativecommons.org/licenses/by/4.0/), which permits unrestricted use, distribution, and reproduction in any medium, provided you give appropriate credit to the original author(s) and the source, provide a link to the Creative Commons license, and indicate if changes were made. The Creative Commons Public Domain Dedication waiver (http://creativecommons.org/publicdomain/zero/1.0/) applies to the data made available in this article, unless otherwise stated. 


\section{Background}

Plants have developed sophisticated structural and chemical mechanisms to resist biotic and abiotic stresses for improving their survival and reproductive fitness. Trichomes, which are protruding epidermal structures on aerial tissues, can protect plants from ultraviolet (UV) light, insect damage, and excess transpiration. Many trichome-borne phytochemicals are involved in resistance against pathogens or in the attraction for pollinators $[1,2]$. Phenylpropanoid-derived phenolics are a large class of plant secondary metabolites actively involved in chemical defenses against herbivores, microbial pathogens, UV-B, and free radicals $[3,4]$. Phenylpropanoid metabolism begins with the synthesis of activated hydroxycinnamic acid ( $p$-coumaroyl-CoA) and the downstream reactions are comprised of the lignin, flavonoid, and other species-specific pathways $[5,6]$. In the lignin pathway, monolignols $p$-coumaryl, coniferyl, and sinapyl alcohols, respectively, produce $\mathrm{H}_{-}$, G-, and S-type lignins. In the flavonoid pathway, dihydroflavonols are produced from $p$-coumaroyl-CoA by the actions of early biosynthetic genes (EBGs) such as CHALCONE SYNTHASE and FLAVANONE 3-HYDROXYLASE. The downstream flavonoid pathway produces catechins, anthocyanins, proanthocyanidins, and other compounds including their glycosides by late biosynthetic genes (LBGs), such as DIHYDROFLAVONOL 4-REDUCTASE (DFR, TT3), LEUKOANTHOCYANIDIN DIOXYGENASE (LDOX, TT18) and ANTHOCYANIDIN SYNTHASE [6].

The molecular mechanism of trichome formation has been characterized extensively in Arabidopsis. Recent studies reveal that there are over 70 genes involved in trichome initiation and development [7, 8]. Among them, a few primary genes encode proteins that form di/tri- protein complexes to regulate the initiation of trichome development [9]. Tri-protein complexes are composed of a R2R3 activator MYB GLABROUS1 (GL1), a bHLH protein GLABROUS3 (GL3) and/or ENHANCER OF GLABRA3 (EGL3) and a WD40 protein TRANSPARENT TESTA GLABRA1 (TTG1), while di-protein complexes do not contain TTG1. These MBW complexes regulate the initiation and patterning of trichomes and several inhibitory R3 MYB proteins such as CAPRICE (CPC) can compete with GL1 to form inactive complexes to inhibit trichome initiation [10]. Similar tri-protein complexes that contain a MYB protein $\mathrm{PRO}-$ DUCTION OF ANTHOCYANIN PIGMENT 1 (PAP1) or TRANSPARENT TESTA 2 (TT2) and TTG1 and TRANSPARENT TESTA 8 (TT8)/GL3 can enhance anthocyanin biosynthesis in Arabidopsis [11, 12] by inducing several late flavonoid biosynthetic genes (LBGs) such as TT3 and TT18 [13]. More recently, transcription of GLABRA2 (GL2) (a homeo-domain transcription factor) was found to be activated by some MBW complexes for trichome production in epidermal cells, which in turn directly represses the expression of MYB75 and TT8, consequently resulting in anthocyanin biosynthesis inhibition in Arabidopsis [14]. These findings clearly indicate anthocyanin production and trichome formation are affected by MBW complexes in Arabidopsis, depending on complex organization $[5,11,15,16]$. Recently, SQUAMOSA PROMOTER BINDING PROTEIN-LIKE 9 (SPL9), a target of miR156, has been shown to disrupt the formation of the MBW complex via interacting competitively with R2R3-MYBs MYB75 to repress anthocyanin accumulation, and SPL9 also inhibits trichome initiation by activating some R3-MYB proteins to compete with GL1 for GL3/EGL3 binding [17].

In the loss-function mutants of the COP9 signalosome (CSN) subunit $5 \mathrm{a}$ and many other subunits, enhanced anthocyanin production and reduced trichome formation [18-21], as well as constitutive photomorphogenic phenotype and altered plant responses to some biotic stresses $[22,23]$, have also been documented. CSN complex consists of structurally interdependent eight subunits (CSN1-CSN8) and is a regulator of cullin-RING ubiquitin E3 ligases (CRLs) [24]. CSN regulates CUL1 ligases (commonly known as SCF complexes), which are involved in auxin signalling [25], flower development [26], jasmonate signalling [22] and gibberellic acid signalling [27] and many other pathways [21, 28, 29]. Therefore, CSN functions as a protease that cleaves RUB1/NEDD8 (both are ubiquitin-like protein covalently attached to CULLINs) in these E3 ligases [22, 24, 30, 31]. This catalytic centre is located in JAMM/MPN motif in CSN5 [20, 32-34]. Recent studies reveal that the function of $\mathrm{SCF}^{\mathrm{CO} 1}$, which is regulated by CSN [35], is involved in repression of anthocyanin accumulation and trichome initiation through the interactions between $\mathrm{SCF}^{\mathrm{COI} 1}$ substrate Jasmonate ZIM-domain (JAZ) proteins and MBW complex components (MYB75, GL1, GL3, EGL3, and TT8) [36]. However, SCF COI1 regulation tends to inhibit both anthocyanin accumulation and trichome initiation simultaneously, which is different from the differential changes in anthocyanin and trichome production found in the null mutants of CSN5a $[18,19]$.

For the purpose to get a deep insight into CSN5a-induced alterations in phenylpropanoid metabolic pathways and trichome production and possible involvement of the TTG1/bHLH/MYB protein complex, characterization of a new Arabidopsis $\operatorname{csn} 5 a$ mutant $s k 372$ isolated from the Saskatoon Arabidopsis T-DNA population was conducted. Defective CSN5a was found to be responsible for enhanced production of numerous phenylpropanoids (including anthocyanins) and carotenoids in this mutant, as well as significantly reduced trichome density and distorted trichome morphology. These phenotypic alterations were closely correlated with enhanced MYB 75 expression and suppressed expression of GL3 and GL2. 


\section{Methods}

\section{Chemicals}

Authentic chemical standards $\beta$-carotene, lutein, $\beta$-cryptoxanthin, zeaxanthin, and violaxanthin were purchased from CaroteNature (www.carotenature.com). Butylated hydroxytoluene (BHT) and ampicillin were purchased from Sigma-Aldrich (www.sigmaaldrich.com/ united-states.html) and corticosterone from Sigma Canada (www.sigmacanada.ca). All solvents used in this study were HPLC grade.

\section{Plant material and growth condition}

Arabidopsis thaliana ecotype Columbia (Col-4) from the Arabidopsis Biological Resource Centre (www.arabidopsis.org) was used to develop the SRC (Saskatoon Research Centre) activation-tagged population [37]. Arabidopsis seeds were cold-treated at $4{ }^{\circ} \mathrm{C}$ for $2-4$ days and then allowed to germinate and grow in soil in a greenhouse at $20{ }^{\circ} \mathrm{C}$ or on $1 / 2$-strength Murashige and Skoog (MS) medium (Sigma, www.sigmaaldrich.com/ canada-english.html) containing $0.8 \%$ agar and $4 \%$ sucrose in a $16 \mathrm{~h}$ light and $8 \mathrm{~h}$ dark regime. For epigenetic studies, the media was supplemented with $500 \mathrm{ng} / \mathrm{mL}$ of the histone deacetylation inhibitor Trichostatin A (TSA) (Sigma T8552) and Arabidopsis seedlings were germinated and grown in the above TSA media for 8 days according to $\mathrm{Xu}$ et al. [38].

\section{Molecular characterization of $s k 372$}

sk372 was selected by screening $\mathrm{T}_{3}$ seeds of the SRC activation-tagged Arabidopsis population by visual examination for pale yellow leaves. The flanking sequences of Arabidopsis genome surrounding the T-DNA insert were isolated and sequenced using the gene-specific primer gwLB2 (Additional file 1: Table S1) and the Universal Genome Walker 2.0 Kit (www.clontech.com) according to the manufacturer's instruction. The obtained sequencing results were analysed using BLASTN against the NCBI non-redundant database to identify the site of T-DNA insertion into the Arabidopsis genome. Southern blot analysis was conducted using $10 \mu \mathrm{g}$ gDNA isolated from sk372 seedlings and overnight digestion with HindIII or Eco RI by following a standard protocol. The T-DNA hybridization probe from pSKI015 and used to analyse the SRC activation-tagged Arabidopsis population was prepared as previously reported [39].

Complementation of $s k 372$ was performed with CSN5a and GL3. Full length native CSN5a coding sequence was amplified from WT seedlings with primer pairs $\mathrm{P} 1+\mathrm{P} 2$ using high fidelity DNA polymerase Pfu Phusion (www.neb.com) (Additional file 1: Table S1). The amplified fragments were sequenced and cloned into $X b a$ I and Sma I sites of the binary vector pBI121 under the control of the CMV35S promoter (35S). Plant expression vector containing 35S: GL3 cassette constructed previously [40] was used for mutant trichome complementation. The constructs were first introduced into Agrobacteria tumefaciens strain GV3101 and then introduced into Arabidopsis by floral dipping [41]. Transformation-positive $\mathrm{T}_{1}$ transgenic plants were detected on $1 / 2$-strength MS media using $50 \mu \mathrm{g} / \mathrm{L}$ kanamycin.

For analyses of mutant heterology and structure of the defective gene in $s k 372$, total RNA was extracted from 14-d seedlings using a commercial RNAEasy mini kit (www.qiagen.com/us/search/rneasy-mini-kit/?akamai-feo =off). Genomic DNA contamination was minimized using on column DNasI digestion as kit manufacturer instructed. cDNA was then obtained using Superscript II reverse transcriptase (www.thermofisher.com/order/ catalog/product/18064014). PCR analysis on the resulting cDNA was performed using the Taq DNA polymerase (www.thermofisher.com/order/catalog/product/18038042). Gene specific primers for CSN5a (Additional file 1: Table S1) were designed based on CSN5a mRNA sequence (AT1G22920). PCR conditions were set up as follows: $94{ }^{\circ} \mathrm{C}$ for $3 \mathrm{~min}, 25$ and 30 cycles at $94{ }^{\circ} \mathrm{C}$ for $30 \mathrm{~s}, 55{ }^{\circ} \mathrm{C}$ for $30 \mathrm{~s}$, and $72{ }^{\circ} \mathrm{C}$ for $1 \mathrm{~min}$, followed at the end by $72{ }^{\circ} \mathrm{C}$ for $5 \mathrm{~min}$.

\section{Analysis of specialized metabolites}

Specialized metabolites in phenylpropanoid pathway were extracted from Arabidopsis seeds in triplicate as described by Lisec et al. [42] and Ayenew et al. [43] with some modifications. Briefly, $50 \mathrm{mg}$ frozen seeds grinded to powder using RETCH-mill (www.retsch.com) and grinding stainless beads. One $\mathrm{mL}$ of pre-chilled extraction solution, methanol/chloroform/water $(2.5 / 1 / 1 \mathrm{v} / \mathrm{v} /$ v), containing internal standards was added. Internal standards of ampicillin ( $1 \mathrm{mg} / \mathrm{mL}$ in water) (www.sigmaaldrich.com/canada-english.html) and corticosterone (1 $\mathrm{mg} / \mathrm{mL}$ in methanol) (www.sigmaaldrich.com/canada-english.html) were used, because they are not Arabidopsis metabolites and detected abundance in the LC-MS/MS is simply from the use of standards and will normalize the machine irregularities and pipetting variations as previously reported [43-45]. The mixture was vortexed and ultra-sonicated for $10 \mathrm{~min}$ each. Following centrifugation at $14000 \mathrm{RPM}$ for $10 \mathrm{~min}$ (at $4{ }^{\circ} \mathrm{C}$ ) the supernatant was collected and mixed with equal volumes of $300 \mu \mathrm{L}$ water and chloroform. The mixtures were vortexed briefly and centrifuged at 14000 RPM for 5 min to collect the upper aqueous phase for LC-MS ${ }^{2}$ analysis. $\mathrm{LC}^{-\mathrm{MS}^{2}}$ analysis was performed using an Agilent 1290 Infinity LC system coupled with a Thermo Q-Exactive Quadrupole-Orbitrap mass spectrometer. Samples were separated with Agilent Eclipse Plus C18 ZORBAX Rapid Resolution High Definition (RRHD) high resolution $1.8 \mu \mathrm{m}$ particle 2.1 i.d. X $50 \mathrm{~mm}$ column as previously 
reported [45]. Detection of metabolites are done in positive and negative electrospray ionization mode (ESI) depending on the metabolites while the internal standards can be detected well in those conditions and to be used for normalization. Metabolites were identified based on mass to charge ratio $(\mathrm{m} / \mathrm{z})$, retention time and fragmentation pattern in comparison to commercial standards, ChemSpider and ReSpect phytochemical databases [43-45].

Carotenoids extracted in triplicate batches from Arabidopsis seed or 14-d seedling leaves were analysed as described by Kormendi et al. [46] with minor modifications. Briefly, $50 \mathrm{mg}$ frozen seeds or leaf tissues were ground using RETCH-mill (Retsch Gmbh, 42,787 Haan, Germany) and extracted twice using $750 \mu \mathrm{L}$ of extraction solvent (2:1:1 hexanes:acetone:ethanol). Following each extraction, samples were mixed in orbital shaker for $5 \mathrm{~min}$ at 1000RPM followed by centrifugation at full speed. Supernatants from both extraction were pooled and dried using a stream of nitrogen gas. The samples were resuspended in $1 \mathrm{~mL}$ solvent mixture of 5:4:1 acetonitrile:dichloromethane:methanol containing $0.5 \%(w / v)$ butylated hydroxytoluene (BHT). Finally, extracts were filtered by $0.2 \mu \mathrm{m}$ nylon syringe filter and placed in a $2 \mathrm{~mL}$ amber HPLC vials. HPLC/UV analysis was conducted using a Hewlett Packard Agilent 1100 chromatograph, a G-7120 diode array detector coupled with Zorbax $\mathrm{C}_{18}$ column $(150 \times 4.6 \mathrm{~mm}, 5 \mu \mathrm{m}$ ID) (www.agilent.com) and HP Chemstation ver. 8.01 software. Sample injection volume of $20 \mu \mathrm{L}$ was used for analysis. Carotenoid identification and quantification were conducted using authentic standard compounds and calibration curves established with these standards as described before [47].

For chlorophyll measurements, 14-day-old seedlings of Col-O and sk372 plants grown on $1 / 2$ MS plus $4 \%$ sucrose [48] was extracted in $80 \%(v / v)$ acetone saturated with $\mathrm{CaCO}_{3}$ in the dark at $20{ }^{\circ} \mathrm{C}$. Anthocyanins were extracted in $1 \%(\mathrm{v} / \mathrm{v}) \mathrm{HCl}$ in methanol. These pigments were assayed in the cleared supernatants after 5 min centrifugation at 10,000 g.The chlorophyll and anthocyanins were quantified according to Mancinelli and Walsh [49].

\section{Microarray analysis}

Total RNAs of Arabidopsis Col-O and sk372 lines were extracted from seedlings grown on 1/2 MS agar plates containing $4 \%$ sucrose for $10 \mathrm{~d}$ and used in the preparation of Cy5- and Cy3-labelled cDNA probes, respectively. An Arabidopsis $26 \mathrm{~K}$ cDNA microarray (Qiagen-Operon Arabidopsis Genome Array Ready Oligo Set Version 1.0, cals.arizona.edu/microarray) was hybridized with Cy5- and Cy3-labelled probe pairs from the Col-O and sk372 samples, respectively. Probe labelling, hybridization and washing were conducted using a CyScribe PostLabelling Kit according to the manufacture's direction (www.selectscience.net/suppliers/amersham-biosciences-corp/ ?compID=2504). Hybridized slides were scanned on a Gene Pix 4000B scanner (www.moleculardevices.com). The intensity (I) of each spot at $\lambda_{546 \mathrm{~nm}}$ and $\lambda_{647 \mathrm{~nm}}$ was transformed into a ratio $\mathrm{I}_{546}: \mathrm{I}_{647}$ with the use of Array-Pro Analyzer 4.0 software (http://www.winsite.com/array/array+pro+analyzer/). Microarray data were initially imported into BASE 1.2.16 and Gene-Spring 6.1 (www.whitelabs.org/Lab\%20Protocols/Genespring/genespring\%2061.pdf) using $\log _{2}$ scale was used to select spots with intensity values $>50$ in at least half of the samples (slides) for further analysis according to Zhao et al. [50]. Data normalization using the per-spot and per-chip Lowess normalization procedure with a smooth factor of 20 and a one-way ANOVA with a parametric test were performed with the value of false discovery rate $(\mathrm{FDR})<0.0001$. A list of genes for which the expression varied by more than 2.0-fold was generated. Gene Ontology (GO) analysis was conducted using the TopGO package [51]. The GO enrichment analysis was performed by selecting both Fisher's exact test and a Kolmogorov-Smirnov like test with $p$-values $<0.05$ and the biological processes of affected GO terms were visualized according to Alexa et al. [51]

\section{Gene transcript analysis using quantitative real time PCR (qPCR)}

RNA aliquots from the microarray experiment were also used in quantitative real-time PCR reactions with SuperScript III First-Strand Synthesis SuperMix and a qRT-PCR kit (www.thermofisher.com/order/catalog/prod uct/11752050) according to the manufacturer's instructions. DNase I was used for on column DNA digestion to minimize genomic DNA contamination. Real-time PCR analysis was carried out using Platinum SYBR Green qPCR SuperMix-UDG kit (www.thermofisher.com/order/ catalog/product/11733046?SID=srch-srp-11733046) on an ABI PRISM StepOnePlus Real-Time PCR System (www.thermofisher.com/order/catalog/product/4376600), following the manufacturer's instructions. Genes ACT2 (AT3G18780) and $E f-1 \alpha$ (AT1G18070) were chosen as endogenous reference genes. For each pair of primers (Additional file 1: Table S1), gel electrophoresis and melting curve analyses were performed to ensure that only a single PCR amplicon of the expected length and melting temperature was generated. All primers used in Real-time RT-PCR analysis are listed in Additional file 2 Table S3. Each sample was assayed in triplicate and data were analysed using the StepOne Software v2.0 (www.advanceduninstaller.com/StepOne-Software-v2_1-bb54a0998d999a0cecd812484ca1bf64-application.htm). The level of each mRNA was calculated using the mean threshold cycle $(\mathrm{Ct})$ value and normalized to that of the reference genes. All results were shown as means of at least three biological replicates with corresponding standard deviations (SD). 


\section{Statistical analysis}

Compositional data were expressed as mean \pm standard error. All measurements were made from triplicate experiments and the data was analysed by ANOVA for significance (LSD at $P<0.05$ ) using SAS 9.0 (SAS Institute, Inc. Cary, NC, USA, www.sas.com/en_us/home.html). For microarray analysis, background-corrected, log ratios of intensity were scaled to have similar distribution across and consistency among arrays. The ANOVA analysis was performed to detect differentially expressed genes using the normalized data [52].

\section{Results}

\section{Phenotypic alterations of the $s k 372$ mutant}

The sk372 mutant was isolated from an Arabidopsis thaliana Columbia (Col-4) T-DNA activation tagged mutant population [37]. Compared to wild-type (WT) Arabidopsis, sk372 exhibited several morphological alterations. Plants grown in different conditions were employed for morphological characterization throughout their life cycle. Plant growth was significantly inhibited at mature and rosette stages and mutant leaves were slightly yellowish (Fig. 1a and b); however, the mutant was capable of producing fertile seeds. Mutant seedlings grown in MS medium with addition of $4 \%$ sucrose had visibly purple stems and cotyledon edges (Fig. 1c). In darkness, WT seedlings showed typical phenotypes of skotomorphogenesis, such as elongated stems, undifferentiated chloroplasts, unexpanded cotyledons, and a germination hook (Fig. 1d), whereas the mutant seedling displayed some phenotypes of photomorphogenesis such as stunted but straight stems without a germination hook and expanded but etiolated cotyledons (Fig. 1d). Moreover, the trichome density observed on the adaxial surface of the fifth true leaf was $3.6 \pm 0.4$ per $\mathrm{mm}^{2}$, while the trichome density in WT was $6.5 \pm 0.4$ (Fig. 1e and $\mathrm{f}$ ). Abnormal trichome size and shapes with fewer trichome branches were noted in the $s k 372$ mutant (Fig. 1g and 1e) compared to WT trichomes (Fig. 1i).

For a better understanding of the observed leaf colour changes in $s k 372$, leaf pigment analysis was conducted. Our data revealed that $s k 372$ leaves had a substantially lower level of chlorophyll but higher levels of total carotenoids and anthocyanin than WT $(p<0.05)$ (Fig. 2). In sk372, the levels of violaxanthin, lutein, and $\beta$-carotene were all significantly higher than their corresponding levels in WT $(\mathrm{p}<0.05)$. The mutant contained slightly higher levels of minor carotenoids zeaxanthin $(0.37 \mu \mathrm{g} / \mathrm{g} . \mathrm{FW})$ and canthaxanthin $(1.36 \mu \mathrm{g} / \mathrm{g} . \mathrm{FW})$ compared to their respective controls $(0.14 \mu \mathrm{g} / \mathrm{g} . \mathrm{FW}$ and $0.39 \mu \mathrm{g} / \mathrm{g} . \mathrm{FW})(p>0.05)$.

\section{Gene expression alteration in $s k 372$}

Additional investigations were undertaken to better understand the molecular mechanisms that lead to the above metabolic alterations in the sk372 mutant. Microarray analysis using 10-day old seedlings, which were grown in MS media with $4 \%$ sucrose and exhibited visible colour changes, suggested that 370 genes were differentially expressed (>2-fold) between WT and sk372 seedlings. A total of $49 \mathrm{GO}$ terms for biological processes were significantly affected, including responses to stimulus, anatomical structure development, and chromosome condensation and organization (Additional file 3: Figure S1). Out of 370 differentially expressed genes (DEGs), 115 suppressed genes coded for proteins that included those related to light reactions, such as the photosystem II $5 \mathrm{KD}$ protein (At1g51400), a putative photosystem II type I chlorophyll a/b binding protein (At1g29910), and the light-harvesting chlorophyll a/b binding protein (At5g54270) (Additional file 4: Table S2). Suppressed genes that were related to sugar metabolism, cell wall and some amino acid biosynthesis were also found. In contrast, 255 genes were enhanced in $s k 372$ seedlings, including genes involved in sugar metabolism as well a gene in the flavonoid pathway (Additional file 2: Table S3). It was noted that in $s k 372$, the enhanced expression of the genes mediating ubiquitin ligase activity was found 2.36.1-fold higher for five RIGN finger protein genes (At1g66040, At5g63780, At4g08590, At4g01270, and At2g42360) and 2.2- 6.4-fold higher for seven F-box protein genes (At4g05470, At5g07610, At5g44980, At5g56810, At3g13680, At1g56240, and At3g59210) compared to WT counterparts. Expression of a putative geranyl diphosphate synthase gene (GPPS) (Atlg78510), functioning upstream of carotenoid biosynthesis, was enhanced in sk372. Moreover, transcription of the UDP-glycosyltransferase gene UGT73C1(At2g36750), specifying glycosylation of cytokinins trans-zeatin and dihydrozeatin [53], was enhanced 7-fold in sk372 seedlings. However, no DEGs (with $\geq 2$-fold change in gene expression) related to trichome development were identified from $s k 372$ in the microarray analysis.

Since MBW (MYB-bHLH-WDR) complexes regulate flavonoid biosynthesis and trichome development [16, 54], we expected that some of the genes associated with these complexes and their regulatory pathways may be differentially expressed. Therefore, further quantitative PCR analyses were conducted to determine the expression levels of trichome development genes, the late stage genes of flavonoid pathways (downstream of dihydroflavonol biosynthesis), and the key genes in the MBW complexes in $s k 372$ and WT. Our data revealed that sk372 exhibited lower transcript levels of the trichome-related homeodomain leucine zipper protein GLABROUS2 (GL2) $(p<0.05)$, but higher levels of TTG1 and GL1 $(p<0.05)$, compared to WT seedlings (Fig. 3a). Moreover, the expression levels of TT2 and the late stage flavonoid synthetic genes TT3 and TT18 were differentially expressed $(p<0.05)$ in the $s k 372$ mutant (Fig. $3 \mathrm{~b})$, with TT3 and TT18 being enhanced 6.4-fold and 1.3-fold, respectively, and TT2 being slightly reduced. 

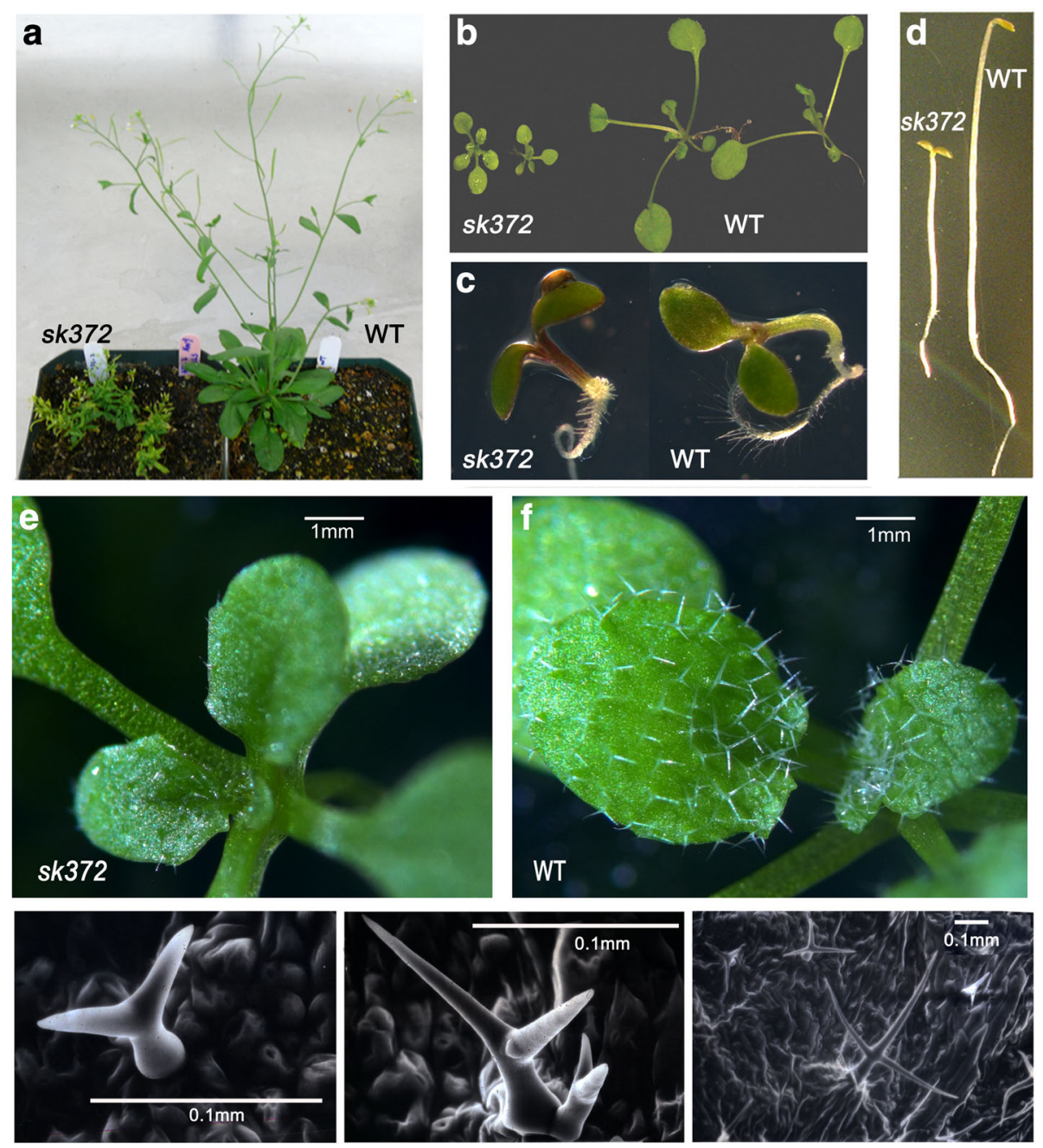

Fig. 1 Morphological alterations of the sk372 mutant compared to wild-type plants. a 45-day old plants; b 17-day old plants; c 4-day old plants in MS media containing 4\% sucrose; $\mathbf{d}$ 4-day old plants grown in the dark; e leaf trichomes of 7-day old sk372 plants; $\mathbf{f}$ leaf trichomes of 7-day old WT plants; $\mathbf{g}$, $\mathbf{h}$ leaf trichomes of 7-day old sk372 plants under scanning electronic microscopy; i leaf trichomes of 7-day old WT plants under scanning electronic microscopy

MYB75 transcription level in $s k 372$ was also higher than in WT $(p<0.05)$.

Since GO term analysis had revealed that chromosome organization genes were affected and the transcript level of bromodomain protein BRD5 (At1g58025), potentially involved in histone acetylation [55] was reduced by 31-fold in sk372 seedlings compared with WT plants the histone deacetylation inhibitor trichostatin A was applied to WT seedlings. This enabled us to examine the effects of histone deacetylation on the transcription of some anthocyanin genes. Our data indicated that expression of MYB75 was enhanced more than 2-fold and that TTG1, TT18 and TT19 (GLUTATHIONE S-TRANSFERASE) were also enhanced $(p<0.05)$ with TSA application (Fig. 3c). Such an increase in the transcripts of all these genes except TT19 was also found in sk372, suggesting the possible involvement of epigenetic regulation of the mutant phenotype expression.

\section{Genetic mutation analysis of $s k 372$}

A genome-walker approach revealed that the T-DNA was inserted in the COP9 SIGNALOSOME 5A (CSN5a) (At1g22920) at the beginning of the fourth exon downstream from the JAMM/MPN motif (Fig. 4a), resulting in a truncated CSN5a, which contained the first 252 amino acid residuals including MPN domain and additional 17 residuals plus a stop codon (LHGSAMSMMVNMEEKKE:*) due to the inserted T-DNA sequence, but lacking of 96 amino acid residuals at the C-terminus from the wild type CSN5a. Homozygosity of the T-DNA insertion in the CSN5a gene of $s k 372$ was confirmed by multiplex PCR using gene-specific primers spanning the full length cDNA and a 


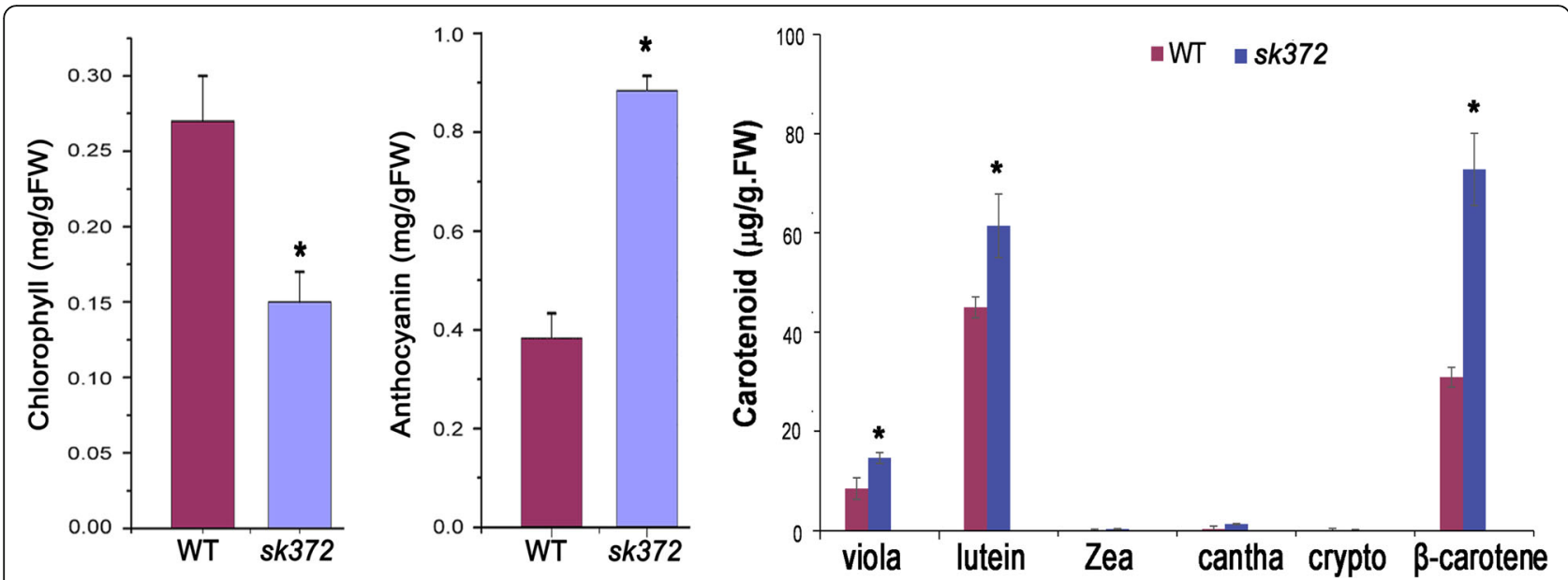

Fig. 2 Leaf pigment abundances in 14-day old plants of sk372 and WT. Significant difference $\left.{ }^{*}\right)(p<0.05)$ in the pigment abundance (total abundance for carotenoids) was found between $s k 372$ and WT. All measurements were made from triplicate experiments and the data was analysed by ANOVA for significance (LSD at $P<0.05)$ using SAS 9.0. viola, violaxanthin; zea, zeaxanthin; cantha, canthaxanthin;

crypto, cryptoxanthin

primer specific to the T-DNA left border (Additional file 5: Figure S2). Southern blot analysis of the $s k 372$ mutant demonstrated a single copy insertion of the T-DNA in the mutant and homozygosity of the T-DNA insertion in the CSN5a gene of $s k 372$ was confirmed by multiplex PCR using gene-specific primers spanning the full-length cDNA and a primer specific to the T-DNA left border (Additional file 5: Figure S2). Reverse transcript-PCR analyses confirmed that the sk372 mutant did not contain the full-length cDNA of CSN5a. Instead, a truncated CSN5a transcript containing the JAMM/MPN motif was detected in the $s k 372$ mutant (Fig. 4b).

\section{Complementation of $s k 372$ with CSN5a}

The $s k 372$ mutant was complemented with the wild type coding sequence of CSN5a driven by the cauliflower mosaic virus 35S (CAMV35S) promoter and transgene expression was enhanced in some complemented T3 offspring (Fig. 5a). Plant morphology was restored in sk372 complemented with the CSN5a transgene (Fig. 5b). Our data clearly indicated that disrupted CSN5a was responsible for the morphological alterations observed in $s k 372$.

\section{Metabolic alteration and restoration in sk372 and the complemented mutant}

Leaf pigment analysis indicated that metabolic alterations occurred in leaves of the mutant. Seed metabolite analysis was also conducted for the purpose to verify whether leaf metabolic changes occurred similarly in seeds, the part of many crops often used as human food and with great significance in agricultural industries for the improvement of crop productivity and nutrition. This analysis might shed light on the potential to practically redirect metabolic pathways for the enforced carotenoids and flavonoids in crop seeds. In $s k 372$ seeds, many metabolites from the pathways of phenylpropanoids and flavonoids were enhanced substantially compared to WT seeds (Fig. $6 \mathrm{a}$ and b) $(p<0.05)$. Strikingly, the levels of 9 metabolites in the late stage of flavonoid biosynthesis increased, including kaempferol and kaempferol glycosides, quercetin glycoside, rutin, peonidin and petunidin (both $O$-methylated anthocyanidins), and isorhamnetin ( $O$-methylated derivative of quercetin). Moreover, substantially higher levels of intermediate compounds in the lignin pathway, including ferulic acid, $O$-methylated coumarin, sinapic acid and its derivatives, were noted in $s k 372$ seeds than WT. Benzoic acid, which can be derived from the phenylpropanoid pathway [56], was also enhanced in $s k 372$. seeds. Moreover, lutein and $\beta$-carotene, dominant carotenoid compounds in Arabidopsis, were enhanced somewhat compared to WT seeds (Fig. 6b). Interestingly, other compounds such as indole-3-carboxylic acid and zeatin-glycoside were also increased in the $s k 372$ mutant. All the alterations in relative abundance were fully or partially restored to varying degrees in the sk372 lines and different CSN5a complementation lines as presented in the heatmap illustration (Fig. 6a). Our data revealed that disruption of CSN5a could lead to metabolic flux redirection in the pathways of seed phenylpropanoids, flavonoids, carotenoids, and even cytokinin glycosylation.

CSN5a overexpression in $s k 372$ was able to restore the entire WT trichome phenotype to the mutant transgenic lines (Fig. 6c). However, overexpression of GL3 alone in the $s k 372$ background (as shown in Additional file 6: Figure S3) resulted in visually increased trichomes in the adaxial surface of the young leaves in $s k 372$ 


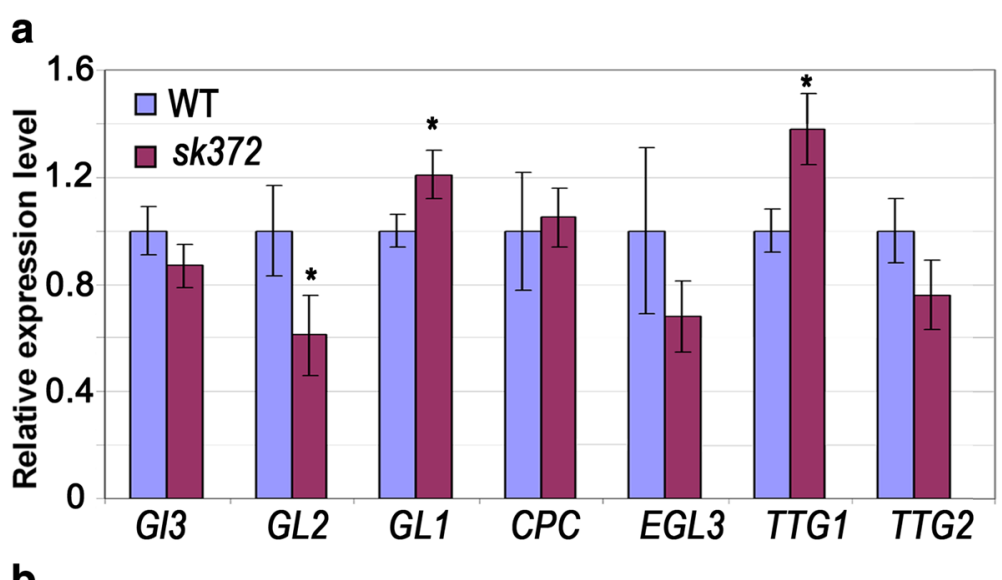

b
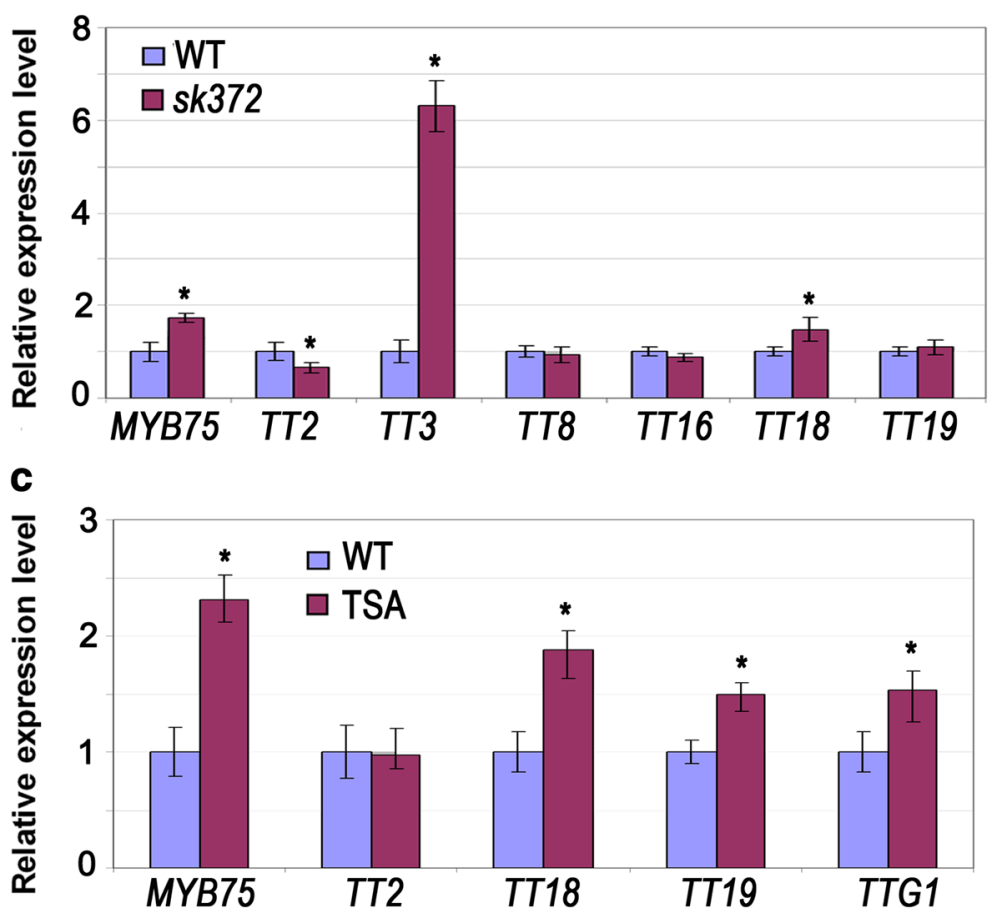

Fig. 3 Transcript levels of genes related to trichome production, flavonoid metabolism, and tri-protein complexes in sk372 mutant relative to WT control. Values for WT were set to an arbitrary number of 1. a Trichome production. b Flavonoid metabolism. c MBW tri-protein complex genes in Arabidopsis seedlings which were germinated and grown for 8 days in the MS media containing $500 \mathrm{ng} / \mathrm{mL}$ of the histone deacetylation inhibitor TSA

complemented lines compared to sk3732 (Fig. 1e). The trichomes produced were largely single pointed (Fig. 6c), not tri-branched as in WT (Fig. If and i).

\section{Discussion}

In this study, characterization of an Arabidopsis mutant $s k 372$ revealed that T-DNA disrupted CSN5 $a$ gene exhibited significant changes in several metabolic pathways, transcriptomic programming, trichome production, and plant morphology, although some similar changes have been documented in $\operatorname{csn} 5 a$ mutants previously [18-21]. In $s k 372$, diverse metabolites including many phenylpropanoids, some carotenoids, and a glycoside of zeatin were found being enhanced while chlorophylls $a$ and $b$ were reduced and the altered expression of numerous genes including some primary genes of the tri-protein (MBW) complexes were revealed.

Microarray analysis combined with a follow-up expression validation using $\mathrm{qRT}-\mathrm{PCR}$ is performed generally for the purpose to reveal possible differences in global gene transcription of tested samples. In this study, our microarray data suggested that in $s k 372$, expression of GPPS (a carotenoid biosynthesis gene) and UGT73C1 (a trans-zeatin glucotransferase gene) was elevated, which was correlated with the enhanced production of carotenoids and trans-zeatin 


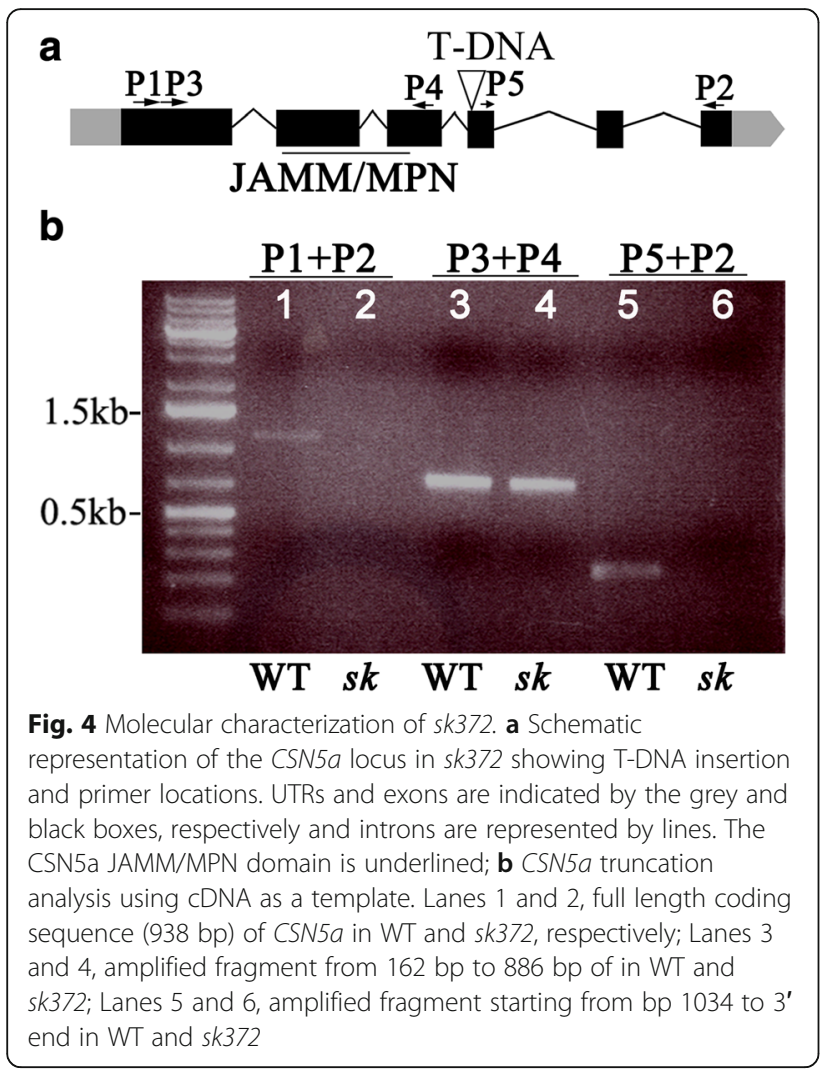

riboside. Such a positive correlation is supported by functional studies on GPPS, a crucial gene upstream in carotenoid pathway by Kirby and Keasling [57] and UGT73C1 (At2g36750), that catalyses glycosylation of trans-zeatin and dihydrozeatin by Hou et al. [53]. Our data also indicated that F-box protein and RING finger protein genes were upregulated in $s k 372$, suggesting that the expression of some genes encoding ligase mediators was also affected due to the defective CSN5a. This is not surprising because CSN5a is crucial for functional regulation of cullin-based ubiquitin E3 ligases $[20,33,34]$ and defective CSN5a might consequently alter the expression of some E3 ligase mediators in sk372.

It was noted that our microarray analysis did not show any genes with altered transcription related to the phenylpropanoid pathway, carotenoid metabolism (except for GPPS), or trichome production between $s k 372$ and WT, even though the non-stringent DEG selection criteria of a 2-fold change was used. We speculated this was due to more subtle differences that were less than the cut-off value of two-fold. Moreover, quantitative PCR is generally considered more sensitive than microarray method [58, 59]. In fact, when we compared transcript levels of key MBW complex genes and some of their downstream targets quantified by qRT-PCR, we noticed that, with the exception of TT3, all were less than 2-fold difference between the WT control and $s k 372$ mutant. One explanation for the subtle differences could be due to the fact that RNA was extracted from whole seedlings and some of the

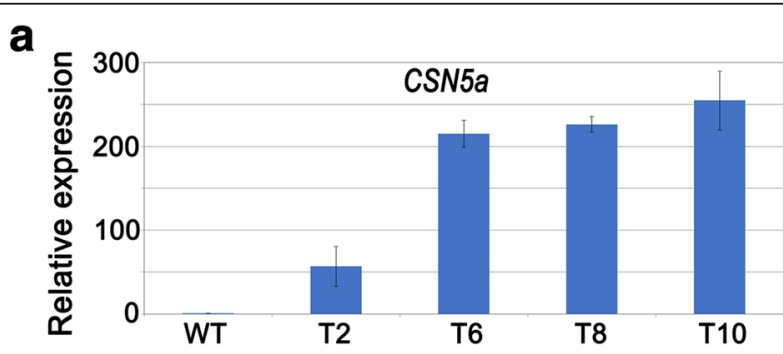

b
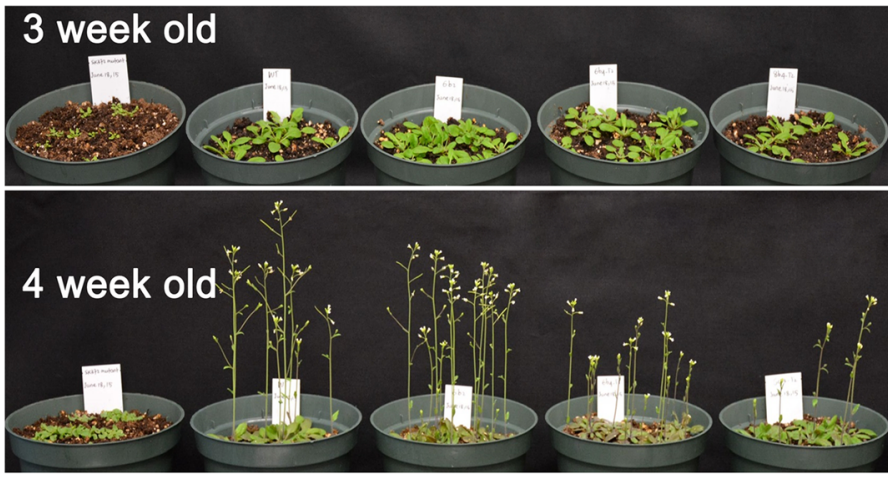

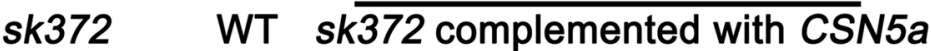

Fig. 5 Genetic complementation of sk372 with CSN5a. a Transgene (CSN5a) expression in the complemented sk372; b Restored phenotypes of the complemented sk372 plants 


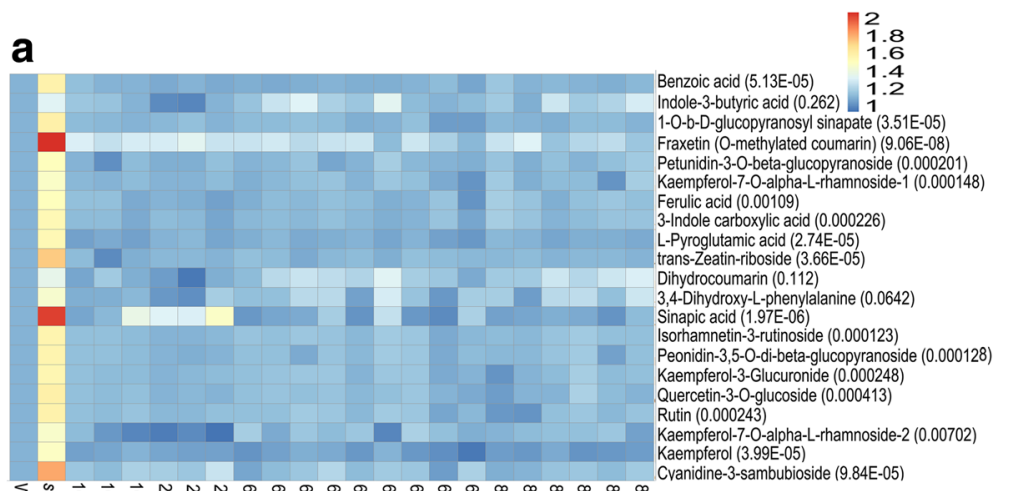

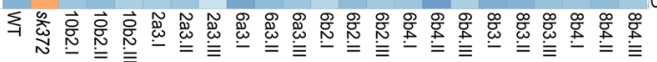

Canidine-3-sambutioside $(9.84 \mathrm{E}-05$

b

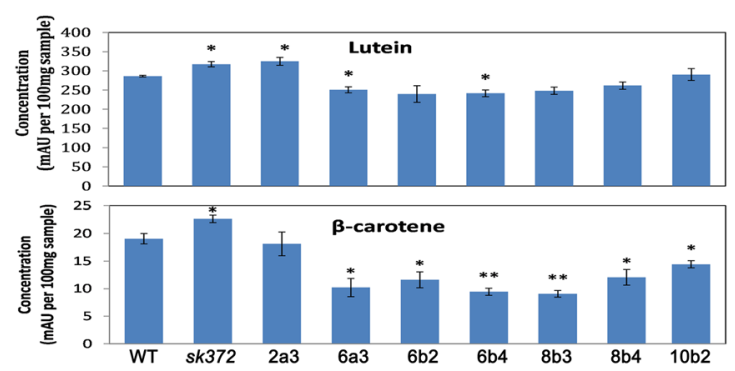

C
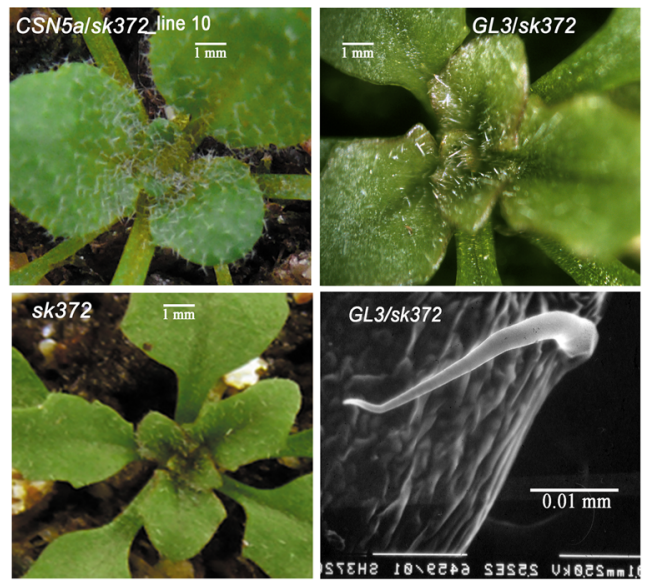

Fig. 6 Alteration of metabolites and trichome development in sk372 and their restoration in the CSN5a complementation lines. a Seed metabolites largely comprising phenylpropanoids and flavonoids. Numbers in the brackets after the compounds in the heatmap are the probabilities of relative abundance difference analysis; $\mathbf{b}$ Seed carotenoid levels, ${ }^{* \prime \prime}$ indicates significantly different from WT, p $<0.05$; $\mathbf{c}$ Close-up examination of seedling trichomes in sk372 complemented with CSN5a or GL3. WT, non transgenic plant of Col-0; sk372, T-DNA activation tagged mutant; 10b21, -II, - III, three biological replicates of CSN5a complementation line 10b2 of T3 generation; 2a3, 6a3, 6b2, 6b4, and 8b3, and 8b4, CSN5a complementation lines of T3 generation. Columns with * and ** were significantly different from WT, $\mathrm{p}<0.05$ and $p<0.01$

genes of interest exhibit tissue-specific expression. For example, GL1 is expressed most strongly in developing trichomes [60], while GL3 transcripts accumulate in both root hairs and trichomes [61].

CSN-regulated anthocyanin accumulation and trichome initiation via $\mathrm{SCF}^{\mathrm{COI} 1}$ occurs through the interaction between the $\mathrm{SCF}^{\mathrm{COI}}$ substrate JAZ and $\mathrm{MBW}$ complex components [36]. Moreover, miR156 enhances anthocyanin and trichome production by suppressing its target SPL9 which destabilizes MBW complexes [17]. However, in $s k 372$, phenylpropanoids including anthocyanin were enhanced while trichome initiation was suppressed, which is different from the simultaneous enhancement or suppression of anthocyanin and trichome production as regulated by miR156-targeted SPL9 and $\mathrm{CSN}$-affected $\mathrm{SCF}^{\mathrm{COI} 1}$. These inconsistent findings suggested that other mechanisms might be involved in sk372. Our qRT-PCR analysis revealed differential 
expression of several primary genes of the MBW complexes in the sk372 mutant. MYB75, TTG1 as well as flavonoid synthetic genes TT3 and TT18 were all enhanced in $s k 372$. Consistently, many phenylpropanoids and their diverse derivatives were found being increased in $s k 372$, in agreement with the finding that enhanced MYB75 can lead to the significantly increased levels of similar phenylpropanoids such as hydroxycinnamic acid esters, and flavonoids, lignin, and anthocyanins that are responsible for the purple colour [62]. Differential expression of the MBW complex genes has been also reported earlier when the expression patterns of these genes were spatially compared in different organs in Arabidopsis [63]. These data together suggested that the MBW complexes, most likely with MYB75 outcompeted over GL1 for bHLH binding in $s k 372$, resulted in the enhanced LBG genes TT3 and TT18 as reported before [13], in favour of the phenylpropanoid biosynthesis.

Moreover, the expression of GL1, which is required for trichome production [64], was also increased in $s k 372$ in which trichome production was actually suppressed, suggesting that GL1 was unlikely responsible for the mutant trichome phenotype. Over-expression of Arabidopsis GL3 alone can result in "hairy canola" with an extremely dense coverage of trichomes on the adaxial leaf surface [40]. In $s k 372$ glabrous adaxial leaf surface and slight but not significant reduction $(p>0.05)$ of $G L 3$ and EGL3 collectively could suggest the possibility that GL3 might be involved in the mutant trichome reduction. However, over-expression of GL3 in sk372 only generated single point trichomes in young leaves and was unable to fully recover trichome production as in WT leaves, suggesting that GL3 alone was not fully responsible for trichome alteration in $s k 372$. In this study, GL2 suppression observed in $s k 372$ due to the MBW complexes predominantly present in $s k 372 \mathrm{might}$ be crucial for the reduced trichome formation and enhanced phenylpropanoid production as found earlier [14]. Moreover, genes coding for the bHLH proteins GL3 and EGL3, GL2, and TTG2 (a WRKY transcription factor) [65] are known being required for trichome production. Possibly significant transcript reduction of GL2 $(p<0.05)$ together with slight but not significant $(p>0.05)$ reduction of the other three genes (GL3, EGL3, and TTG2) could collectively involve in the trichome phenotypes in sk372. In addition, cytokinin (6-benzylaminopurine, BAP) is known being able to induce trichome production in Arabidopsis [66]. Our metabolic profile indicated that in $s k 372$ glycoside of trans-zeatin abundance was increased. Concomitantly the level of active zeatin in sk372 was likely decreased, which might lead to the suppressed trichome formation in the mutant. Further studies are required to validate these hypotheses. Moreover, the enhanced TTG1 in $s k 372$ could be related to the enhanced phenylpropanoids, but unlikely to be responsible for neither the trichome density reduction nor for trichome distortion and altered flavonoid patterns, for ttg1 mutants typically had few or no trichomes and significantly reduced anthocyanins as reviewed by Broun [11]. It would be interesting to verify the effects of altered expression of the MBW complex components on flavonoid and trichome production in $s k 372$.

Moreover, the application of histone deacetylase inhibitor TSA induced the expression of MBW complex genes MYB75 and TTG1, in addition to TT18 and TT19, and slightly suppressed TT2 expression in the Arabidopsis WT plants $(p<0.05)$, as found similarly in the mutant of $s k 372$ in which slightly enhanced TT19 and significantly suppressed TT2 $(\mathrm{p}<0.05)$ were noted. These findings indicated that histone acetylation is involved in the regulation of the root hair patterning in Arabidopsis seedlings according to $\mathrm{Xu}$ et al. [38]. Additionally, the bromodomain protein GTE6 is known to control Arabidopsis leaf development through histone acetylation/deacetylation regulation [67]. Possibility could not be excluded that downregulation of the bromodomain protein gene $B R D 5$ revealed by microarray analysis in this study might be involved in the epigenetic regulation of the observed phenotypes in $s k 372$.

CSN5a is a crucial subunit of the COP9 signalosome (CSN) and removal of RUB1/NEDD8 is performed by CSN5a via its protease activity maintained in JAMMAN domain $[20,33,34]$. CSN5a can also stably exist independent of the CSN in vivo and function actively either as part of the CSN holocomplex or independently [24]. In $s k 372$, T-DNA was inserted at the site downstream of JAMM/MPN domain (Fig. 5a), likely maintaining the crucial domain in the truncated CSN5a in sk372. Slightly difference in phenotypes in the two Arabidopsis mutants $\operatorname{csn} 5 a-1$ (T-DNA inserted immediately downstream of ATG start condon) and $\operatorname{csn} 5 a-2$ (T-DNA inserted downstream of JAMM/MPN) were documented by Gusmaroli et al., [20]. It would be interesting to compare the morphological, chemical and transcriptomic changes in sk372 with those in the above two mutants and try to find out how the remaining domains in the truncated CSN5a in $s k 372$ affected the function of independent form alone and CSN complexes.

\section{Conclusions}

Defective CSN5a in $s k 372$ resulted in significant changes in the metabolism of a wide range of phenylpropanoid and carotenoid compounds as well as hormones. These changes in flavonoid and trichome production were likely the consequences of altered transcription of genes associated with the of TTG1/bHLH/MYB complex. 


\section{Additional files}

Additional file 1: Table S1. Primers used in this study. (XLSX $10 \mathrm{~kb}$ )

Additional file 2: Figure S1. Overrepresented categories of enriched gene ontology based on differential gene expression between sk372 seedlings compared with wild type Arabidopsis. Circle sizes represent larger or smaller numbers of differentially expressed genes. Circle colours represent $p$ values. (TIF $1781 \mathrm{~kb}$ )

Additional file 3: Table S2. Down-regulated gene expression more than 2-fold in sk372 compared to wild-type control. (XLSX 25 kb)

Additional file 4: Table S3. Up-regulated gene expression more than 2 fold in sk372 compared to wild-type control. (XLSX $31 \mathrm{~kb}$ )

Additional file 5: Figure S2. Molecular characterization of $s k 372$. (A) Southern blot; (B) homozygous determination of sk372. (D) CSN5a truncation analysis using CDNA as a template. Lanes 1 and 2, full length (938 bp) of CSN5a in WT and sk372, respectively; Lanes 3 and 4, amplified fragment from 162 bp to 886 bp in WT and sk372; Lanes 5 and 6, amplified fragment starting from bp 1034 to $3^{\prime}$ end in WT and sk372.The presence of transgene GL3 in the different independent lines of transgenic sk372. M, $1 \mathrm{~kb}$ plus DNA marker (Invitrogen); T1-T8, different independent lines of transgenic plants; WT, wild type control. (TIF 7690 kb)

Additional file 6: Figure S3. The presence of transgene GL3 in the different independent lines of 563 transgenic sk372. M, 1 kb plus DNA marker (Invitrogen); T1-T8, different independent lines of 564 transgenic plants; WT, wild type control. (TIF $879 \mathrm{~kb}$ )

\section{Abbreviations}

\section{Plant Materials}

Col-4: Columbia ecotype-4 of Arabidopsis thaliana; sk372: An Arabidopsis TDNA activation-tagged mutant selected from of the SRC (Saskatoon Research Centre) population; WT: Wild type plants

\section{Protein complex components and related proteins}

bHLH: Basic helix-loop-helix; BRD5: A bromodomain protein; COP9: Constitutive photomorphogenic9; CPC: CAPRICE; CSN5a: COP9 signalosome subunit 5a; EGL3: ENHANCER OF GLABRA3; GL1: GLABRA1; GL2: GLABRA2; GL3: GLABRA3; GPPS: Geranyl diphosphate synthase; JAMM: Jab1/MPN/MOV34 domain; MBW: MYB-bHLH-WDR complexes; MPN: MPR1p and PAD1p amino-terminal; MYB: Myeloblastosis family of transcription factors; TT: Transperant Testa; TTG1: TRANSPARENT TESTA GLABRA1; UGT: UDP-glycosyltransferase

\section{Chemicals and Bioinformatics and chemical analysis}

BHT: Butylated hydroxytoluene; DEGs: Differentially expressed genes; GO: Gene Ontology; HPLC: High-performance liquid chromatography; LCMS $^{2}$ : Liquid chromatography-tandem mass spectrometry; MS: Murashige and Skoog medium for tissue culture; Q-PCR: Real-time quantitative PCR; TSA: Trichostatin A, a histone deacetylation inhibitor; UV: Ultraviolet light

\section{Acknowledgments}

Authors acknowledged the technical help from Drs. Guo-Feng Liu and FuMing Wang at the Anhui Agricultural University for data analysis and growth chamber management of sk372.

\section{Funding}

National Natural Science Foundation of China (Award numbers 31770734 and 31370687).

\section{Availability of data and materials}

All data generated or analysed during this study are included in this published article and its Additional files 1, 2, 3, 4, 5 and 6.

\section{Authors' contributions}

SW isolated and characterized sk372 together with XL. Both together prepared manuscript draft. BAF and LA completed metabolic and gene expression profiling of $\mathrm{T} 2$ transgenic plants and revised manuscript. MYG and $\mathrm{AH}$ conceived the study and provided a critical suggestion. All authors read and approved the final manuscript.
Ethics approval and consent to participate

Not applicable.

\section{Consent for publication}

Not applicable.

\section{Competing interests}

The authors declare that they have no competing interests.

\section{Publisher's Note}

Springer Nature remains neutral with regard to jurisdictional claims in published maps and institutional affiliations.

\section{Author details}

${ }^{1}$ State Key Laboratory of Tea Plant Biology and Utilization, Anhui Agricultural University, Hefei, Anhui, China. ${ }^{2}$ Agriculture and Agri-Food Canada, Saskatoon Research Center, Saskatoon, SK, Canada. ${ }^{3}$ Department of Biochemistry and Biomedical Sciences, Faculty of Health Sciences, McMaster University, Hamilton, ON, Canada. ${ }^{4}$ Agriculture and Agri-Food Canada and Department of Biology, University of Western Ontario, London, ON, Canada.

Received: 25 April 2018 Accepted: 7 June 2018

Published online: 25 June 2018

\section{References}

1. Wagner GJ. Secreting glandular trichomes: more than just hairs. Plant Physiol. 1991;96:675-9.

2. Wagner GJ, Wang E, Shepherd RW. New approaches for studying and exploiting an old protuberance, the plant trichome. Ann Bot. 2004;93:3-11.

3. Cheynier V, Comte G, Davies KM, Lattanzio V, Martens S. Plant phenolics: recent advances on their biosynthesis, genetics, andecophysiology. Plant Physiol Biochem. 2013;72:1-20.

4. Shalaby S, Horwitz BA. Plant phenolic compounds and oxidative stress: integrated signals in fungal-plant interactions. Curr Genet. 2015;61:347-57.

5. Chezem WR, Clay NK. Regulation of plant secondary metabolism and associated specialized cell development by MYBs and bHLHs. Phytochemistry. 2016;131:26-43.

6. Vogt T. Phenylpropanoid biosynthesis. Mol Plant. 2010;3:2-20

7. Taheri A, Gao P, Yu M, Cui D, Regan S, Parkin I, et al. A landscape of hairy and twisted: hunting for new trichome mutants in the Saskatoon Arabidopsis T-DNA population. Plant Biol. 2015;17:384-94.

8. Marks MD, Wenger JP, Gilding E, Jilk R, Dixon RA. Transcriptome analysis of Arabidopsis wild-type and gl3-sst Sim trichomes identifies four additional genes required for trichome development. Mol Plant. 2009;2:803-22.

9. Ishida T, Kurata T, Okada K, Wada T. A genetic regulatory network in the development of trichomes and root hairs. Annu Rev Plant Biol. 2008;59:365-86

10. Pesch $M$, Hülskamp M. One, two, three...models for trichome patterning in Arabidopsis? Curr Opin Plant Biol. 2009;12:587-92.

11. Broun P. Transcriptional control of flavonoid biosynthesis: a complex network of conserved regulators involved in multiple aspects of differentiation in Arabidopsis. Curr Opin Plant Biol. 2005:8:272-9.

12. Zhou LL, Shi MZ, Xie DY. Regulation of anthocyanin biosynthesis by nitrogen in TTG1-GL3/TT8-PAP1-programmed red cells of Arabidopsis thaliana. Planta. 2012;236:825-37.

13. Li S. Transcriptional control of flavonoid biosynthesis Fine-tuning of the MYB-bHLH-WD40 (MBW) complex. Plant Signal Behav. 2014;9:e27522.

14. Wang X, Wang X, Hu Q, Dai X, Tian H, Zheng K, et al. Characterization of an activation-tagged mutant uncovers a role of GLABRA2 in anthocyanin biosynthesis in Arabidopsis. Plant J. 2015;83:300-11.

15. Liu M, Tian $H$, Wu J, Cang R, Wang R, Qi X, et al. Relationship between gene expression and the accumulation of catechin during spring and autumn in tea plants (Camellia sinensis L.). Hortic Res. 2015;2:15011.

16. Xu W, Dubos C, Lepiniec L. Transcriptional control of flavonoid biosynthesis by MYB-bHLH-WDR complexes. Trends Plant Sci. 2015;20:176-85.

17. Gou J-Y, Felippes FF, Liu C-J, Weigel D, Wang J-W. Negative regulation of anthocyanin biosynthesis in Arabidopsis by a miR156-targeted SPL transcription factor. Plant Cell. 2011:23:1512-22.

18. Dohmann EMN, Kuhnle C, Schwechheimer C. Loss of the CONSTITUTIVE PHOTOMORPHOGENIC9 signalosome subunit 5 is sufficient to cause the cop/det/fus mutant phenotype in Arabidopsis. Plant Cell. 2005;17:1967-78. 
19. Gusmaroli G, Feng S, Deng XW. The Arabidopsis CSN5A and CSN5B subunits are present in distinct COP9 signalosome complexes, and mutations in their JAMM domains exhibit differential dominant negative effects on development. Plant Cell. 2004;16:2984-3001.

20. Gusmaroli G, Figueroa P, Serino G, Deng XW. Role of the MPN subunits in COP9 signalosome assembly and activity, and their regulatory interaction with Arabidopsis Cullin3-based E3 ligases. Plant Cell. 2007;19:564-81.

21. Stratmann JW, Gusmaroli G. Many jobs for one good COP - the COP9 signalosome guards development and defense. Plant Sci. 2012;185-186:50-64.

22. Hind SR, Pulliam SE, Veronese P, Shantharaj D, Nazir A, Jacobs NS, et al. The COP9 signalosome controls jasmonic acid synthesis and plant responses to herbivory and pathogens. Plant J. 2011;65:480-91.

23. Liu Y, Schiff M, Serino G, Deng X-W, Dinesh-Kumar SP. Role of SCF ubiquitin-ligase and the COP9 signalosome in the $\mathrm{N}$ gene-mediated resistance response to tobacco mosaic virus. Plant Cell. 2002;14:1483-96.

24. Wei N, Serino G, Deng XW. The COP9 signalosome: more than a protease. Trends Biochem Sci. 2008;33:592-600.

25. Schwechheimer C, Serino G, Callis J, Crosby WL, Lyapina S, Deshaies RJ, et al. Interactions of the COP9 signalosome with the E3 ubiquitin ligase SCFTIRI in mediating auxin response. Science. 2001;292:1379-82.

26. Wang X, Feng S, Nakayama N, Crosby WL, Irish V, Deng XW, et al. The COP9 signalosome interacts with SCF(UFO) and participates in Arabidopsis flower development. Plant Cell. 2003;15:1071-82.

27. Dohmann EMN, Nill C, Schwechheimer C. DELLA proteins restrain germination and elongation growth in Arabidopsis thaliana COP9 signalosome mutants. Eur J Cell Biol. 2010;89:163-8.

28. Azevedo C, Sadanandom A, Kitagawai K, Freialdenhoven A, Shirasu K, Schulze-Lefert P. The RAR1 interactor SGT1, an essential component of R gene-triggered disease resistance. Science. 2002;295:2073-6.

29. Stone SL, Anderson EM, Mullen RT, Goring DR. ARC1 is an E3 ubiquitin ligase and promotes the ubiquitination of protein during the rejektion of selt-incompatible Brassica pollen. Plant Cell. 2003;15:885-98.

30. Wee S, Geyer RK, Toda T, D a W. CSN facilitates Cullin-RING ubiquitin ligase function by counteracting autocatalytic adapter instability. Nat Cell Biol. 2005;7:387-91.

31. Jin D, Li B, Deng XW, Wei N. Plant COP9 signalosome subunit 5, CSN5. Plant Sci. 2014;224:54-61.

32. Cope GA, Suh GSB, Aravind L, Schwarz SE, Zipursky SL, Koonin EV, et al. Role of predicted metalloprotease motif of Jab1/Csn5 in cleavage of Nedd8 from Cul1. Science. 2002;298:608-11.

33. Lyapina S, Cope G, Shevchenko A, Serino G, Tsuge T, Zhou C, et al. Promotion of NEDD8-CUL1 conjugate cleavage by COP9 signalosome. Science. 2001;292:1382-5.

34. Wei N, Deng XW. The COP9 signalosome. Annu Rev Cell Dev Biol. 2003;19:261-86.

35. Feng S, Ma L, Wang X, Xie D, Dinesh-Kumar SP, Wei N, et al. The COP9 signalosome interacts physically with SCF COI1 and modulates jasmonate responses. Plant Cell. 2003;15:1083-94.

36. Qi T, Song S, Ren Q, Wu D, Huang H, Chen Y, et al. The Jasmonate-ZIMdomain proteins interact with the WD-repeat/bHLH/MYB complexes to regulate Jasmonate-mediated anthocyanin accumulation and Trichome initiation in Arabidopsis thaliana. Plant Cell. 2011:23:1795-814.

37. Robinson SJ, Tang LH, Mooney BA, McKay SJ, Clarke WE, Links MG, et al. An archived activation tagged population of Arabidopsis thaliana to facilitate forward genetics approaches. BMC Plant Biol. 2009;9:101.

38. Xu C-R, Liu C, Wang Y-L, Li L-C, Chen W-Q, Xu Z-H, et al. Histone acetylation affects expression of cellular patterning genes in the Arabidopsis root epidermis. Proc Natl Acad Sci U S A. 2005;102:14469-74.

39. Wei S, Xi YZ, Song DP, Wei H, Gruber MY, Gao MJ, et al. Quantitative and structural analyses of T-DNA tandem repeats in transgenic Arabidopsis SK mutant lines. Plant Cell Tissue Organ Cult. 2015;123:183-92.

40. Gruber MY, Wang S, Ethier S, Holowachuk J, Bonham-Smith PC, Soroka J, et al. "HAIRY CANOLA" - Arabidopsis GL3 induces a dense covering of trichomes on Brassica napus seedlings. Plant Mol Biol. 2006;60:679-98.

41. Clough SJ, Bent AF. Floral dip: a simplified method for Agrobacteriummediated transformation of Arabidopsis thaliana. Plant J. 1998;16:735-43.

42. Lisec J, Schauer N, Kopka J, Willmitzer L, Fernie AR. Gas chromatography mass spectrometry-based metabolite profiling in plants. Nat Protoc. 2006;1:387-96.

43. Ayenew B, Degu A, Manela N, Perl A, Shamir MO, Fait A. Metabolite profiling and transcript analysis reveal specificities in the response of a berry derived cell culture to abiotic stresses. Front Plant Sci. 2015;6:728.
44. Degu A, Ayenew B, Cramer GR, Fait A. Polyphenolic responses of grapevine berries to light, temperature, oxidative stress, abscisic acid and jasmonic acid show specific developmental-dependent degrees of metabolic resilience to perturbation. Food Chem. 2016;212:828-36.

45. Hochberg U, Degu A, Cramer GR, Rachmilevitch S, Fait A. Cultivar specific metabolic changes in grapevines berry skins in relation to deficit irrigation and hydraulic behavior. Plant Physiol Biochem. 2015;88:42-52.

46. Kormendi A, Amyot L, Omari K, McDowell T, Hannoufa A. A fast, adaptable piecewise gradient method for high-throughput quantification of leaf carotenoids using RP-HPLC-PDA. Anal Methods. 2016;8:4955-64.

47. Wei S, Li X, Gruber MY, Li R, Zhou R, Zebarjadi A, et al. RNAi-mediated suppression of DET1 alters the levels of carotenoids and sinapate esters in seeds of Brassica napus. J Agric Food Chem. 2009;57:5326-33.

48. Baier M, Hemmann G, Holman R, Corke F, Card R, Smith C, et al. Characterization of mutants in Arabidopsis showing increased sugar-specific gene expression, growth, and developmental responses. Plant Physiol. 2004;134:81-91.

49. Mancinelli AL, Walsh L. Photocontrol of anthocyanin synthesis. Plant Physiol. 1979;63:841-6.

50. Zhao J, Buchwaldt L, Rimmer SR, Sharpe A, Mcgregor L, Bekkaoui D, Hegedus D. Patterns of differential gene expression in Brassica napus cultivars infected with Sclerotinia sclerotiorum. Mol Plant Pathol. 2009;10:635-49.

51. Alexa A, Rahnenführer J, Lengauer T. Improved scoring of functional groups from gene expression data by decorrelating GO graph structure. Bioinformatics. 2006;22:1600-7.

52. Zhao J, Wang J, An L, Doerge RW, Chen ZJ, Grau CR, et al. Analysis of gene expression profiles in response to Sclerotinia sclerotiorum in Brassica napus. Planta. 2007;227:13-24

53. Hou B, Lim EK, Higgins GS, Bowles DJ. N-glucosylation of cytokinins by glycosyltransferases of Arabidopsis thaliana. J Biol Chem. 2004;279:47822-32.

54. Pattanaik S, Patra B, Singh SK, Yuan L. An overview of the gene regulatory network controlling trichome development in the model plant, Arabidopsis. Front Plant Sci. 2014;5:259.

55. Pandey R, Müller A, Napoli CA, Selinger DA, Pikaard CS, Richards EJ, et al. Analysis of histone acetyltransferase and histone deacetylase families of Arabidopsis thaliana suggests functional diversification of chromatin modification among multicellular eukaryotes. Nucleic Acids Res. 2002;30:5036-55.

56. Widhalm JR, Dudareva N. A familiar ring to it: biosynthesis of plant benzoic acids. Mol Plant. 2015;8:83-97.

57. Kirby J, Keasling JD. Biosynthesis of plant isoprenoids: perspectives for microbial engineering. Annu Rev Plant Biol. 2009;60:335-55.

58. Wang T, Brown MJ. mRNA quantification by real time TaqMan polymerase chain reaction: validation and comparison with RNase protection. Anal Biochem. 1999;269:198-201.

59. Bustin SA. Absolute quantification of mRNA using real-time reverse transcription polymerase chain reaction assays. J Mol Endocrinol. 2000; 25:169-93.

60. Larkin JC, Oppenheimer DG, Pollock S, Marks MD. Arabidopsis GLABROUS1 gene requires downstream sequences for function. Plant Cell. 1993:5:1739-48.

61. Schiefelbein J, Huang L, Zheng X. Regulation of epidermal cell fate in Arabidopsis roots: the importance of multiple feedback loops. Front Plant Sci. 2014;5:47. https://doi.org/10.3389/fpls.2014.00047.

62. Borevitz JO, Xia Y, Blount J, Dixon RA, Lamb C. Activation tagging identifies a conserved MYB regulator of phenylpropanoid biosynthesis. Plant Cell. 2000;12:2383.

63. Petroni $\mathrm{K}$, Tonelli $\mathrm{C}$. Recent advances on the regulation of anthocyanin synthesis in reproductive organs. Plant Sci. 2011;181:219-29.

64. Oppenheimer DG, Herman PL, Sivakumaran S, Esch J, Marks MD. A myb gene required for leaf trichome differentiation in Arabidopsis is expressed in stipules. Cell. 1991:67:483-93.

65. Ishida T, Hattori S, Sano R, Inoue K, Shirano Y, Hayashi H, et al. Arabidopsis TRANSPARENT TESTA GLABRA2 is directly regulated by R2R3 MYB transcription factors and is involved in regulation of GLABRA2 transcription in epidermal differentiation. Plant Cell. 2007;19:2531-43.

66. Maes L, Inze D, Goossens A. Functional specialization of the TRANSPARENT TESTA GLABRA1 network allows differential hormonal control of laminal and marginal trichome initiation in Arabidopsis rosette leaves. Plant Physiol. 2008;148:1453-64.

67. Chua YL, Watson LA, Gray JC. The transcriptional enhancer of the pea plastocyanin gene associates with the nuclear matrix and regulates gene expression through histone acetylation. Plant Cell. 2003;15:1468-79. 